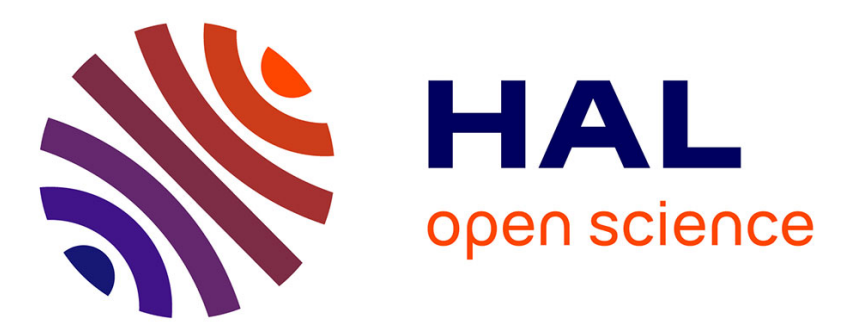

\title{
Structure, Dynamics, and Thermodynamics of Intruded Electrolytes in ZIF-8
}

\author{
Guillaume Fraux, Anne Boutin, Alain Fuchs, François-Xavier Coudert
}

\section{To cite this version:}

Guillaume Fraux, Anne Boutin, Alain Fuchs, François-Xavier Coudert. Structure, Dynamics, and Thermodynamics of Intruded Electrolytes in ZIF-8. Journal of Physical Chemistry C, 2019, 123 (25), pp.15589-15598. 10.1021/acs.jpcc.9b02718 . hal-02168637

\section{HAL Id: hal-02168637 https://hal.science/hal-02168637}

Submitted on 28 Jun 2019

HAL is a multi-disciplinary open access archive for the deposit and dissemination of scientific research documents, whether they are published or not. The documents may come from teaching and research institutions in France or abroad, or from public or private research centers.
L'archive ouverte pluridisciplinaire $\mathbf{H A L}$, est destinée au dépôt et à la diffusion de documents scientifiques de niveau recherche, publiés ou non, émanant des établissements d'enseignement et de recherche français ou étrangers, des laboratoires publics ou privés. 


\title{
Structure, Dynamics and Thermodynamics of Intruded Electrolytes in ZIF-8
}

\author{
Guillaume Fraux, ${ }^{\dagger}$ Anne Boutin,$^{\ddagger}$ Alain H. Fuchs, ${ }^{\dagger}$ and Franccois-Xavier \\ Coudert*, \\ †Chimie ParisTech, PSL University, CNRS, Institut de Recherche de Chimie, Paris, 75005 \\ Paris, France \\ $\ddagger$ †ASTEUR, Département de Chimie, École normale supérieure, PSL University, Sorbonne \\ Université, CNRS, 75005 Paris, France \\ E-mail: fx.coudert@chimieparistech.psl.eu
}




\begin{abstract}
We report here the properties of $\mathrm{LiCl}$ aqueous solutions at various concentrations confined inside the pores of the ZIF-8 metal-organic framework, based on classical molecular dynamics simulations. This system has been proposed for applications in the storage or dissipation of mechanical energy, using the liquid-phase intrusion of concentrated electrolytes in the hydrophobic framework. We describe the structure of the liquids and the influence of confinement, their dynamics, the mechanical properties of ZIF-8 and the impact of liquid intrusion on them. We show that the presence of the electrolyte has a moderate impact on the ZIF-8 framework, while the presence of the ZIF-8 matrix strongly influences the behavior of the confined aqueous solution, affecting the overall properties of the system. We also computed the free energy profile for the entry of water molecules and ions into the nanopores, showing a difference between anions and cations.
\end{abstract}




\section{Introduction}

Nanoporous crystalline materials such as zeolites, metal-organic frameworks (MOF), carbon nanotubes and inorganic open frameworks enjoy a wide range of applications, ranging from catalysis, fluid separation and purification, to gas capture and detection of dangerous molecules. The fundamental basis for most of these applications is the material's capability to adsorb large quantities of molecules inside its nanometer-sized pores, due to its large specific surface area.$^{1}$ In this context, hydrophobic nanoporous materials offer the advantage that the uptake of water from the gas phase - e.g., humidity in the air — is very small. ${ }^{24}$ Given that water is often strongly adsorbed and competes with other molecules for adsorption sites, hydrophobic molecular sieves can offer higher separation properties. $\frac{516}{6}$

One of the novel applications that has been proposed for hydrophobic nanoporous materials is related to mechanical energy storage or dissipation through water intrusion. ${ }^{\frac{78}{7}}$ In a hydrophobic porous material, the pressure at which external water will enter the pore space is greater than the vapor pressure of water. That is, adsorption happens only in the liquid phase - and this high-pressure adsorption is called intrusion. ${ }^{9}$ This phenomenon has been extensively studied in inorganic nanoporous materials, such as zeolites, $\frac{10}{13}$ and more recently evidenced in hydrophobic metal-organic frameworks. ${ }^{14}[16$ Depending on the nature of the nanoporous material and the strength of the host-guest interactions, intrusion curves can have different shapes and be classified as either as a molecular spring, a shock adsorber, or a bumper. This classification depends on the level of hysteresis during an intrusion-extrusion cycle. For more details, we refer the interested reader to a review we recently wrote. ${ }^{9}$

A very sought-after property related to the intrusion of water in hydrophobic frameworks is the ability to tune the intrusion pressure and the amount of hysteresis present. This can be achieved by chemical modifications of the structure of the host material, $\frac{17}{17}$ or by changing the nature of the liquid - namely, by adding ions to the water. $\frac{18}{18}$ Depending on the size of the ions and porous channels, in some cases only water can enter the nanopores while ions stay 
in the bulk liquid. The change in the intrusion pressure is then directly related to additional osmotic pressure the fluid has to overcome to enter the structure. ${ }^{\frac{16}{6}}$ But in other cases, the addition of ions has a more complex impact on both the intrusion pressure and the shape of intrusion curves. For example, in the pure-silica analogue of the $\beta$ zeolite, ${ }^{19}$ increasing the electrolyte concentration from $5 \mathrm{~mol} / \mathrm{L}$ to $10 \mathrm{~mol} / \mathrm{L}$ only shifts the intrusion pressure, but increasing it again to $15 \mathrm{~mol} / \mathrm{L}$ changes the overall behavior from a shock-absorber to a spring. ${ }^{20}$ Such changes indicate that the interaction between the electrolyte fluid and the host structure is not merely reduced to a simple effect of size-based exclusion. At the same time, in situ X-ray diffraction measurements performed during intrusion-extrusion cycles showed that $\mathrm{MgCl}_{2}$ ions can enter a pure-silica ferrierite during intrusion. ${ }^{21]}$ All this evidence points to a more complex effect than pure osmotic pressure when using an electrolyte fluid for intrusion.

Moreover, we know that adsorption of water in the gas phase can induce large structural changes in nanoporous materials. ${ }^{2223}$ This is particular true of soft porous crystals, ${ }^{24}$ such as flexible MOFs, $\frac{25}{25}$ exhibiting dynamic frameworks that are able to respond to external stimuli. This has been studied, by both experimental and computational means, on the MIL-53 family of "breathing" frameworks: the presence of water influences the structure of MIL-53(Cr), 26 and is responsible for the occurrence of numerous structural transitions in MIL-53(Ga), as a function of both water vapor pressure and temperature. ${ }^{[27 / 28}$ Relatively little is known, in contrast, on the impact of liquid water - and aqueous solution — intrusion on the structure of flexible MOFs.

Here, we are interested in the study of the high-pressure electrolyte intrusion in ZIF-8. ZIF-8 is a nanoporous material part of the Zeolitic Imidazolate Frameworks (ZIF) family. These MOF are built from imidazolate anions as their organic linker $(\mathrm{mim}=2$ methylimidazolate in the case of ZIF-8) and a divalent metal cation (here, $\mathrm{Zn}^{2+}$ ). The frameworks of ZIFs are four-connected, meaning that the ZIFs adopt zeolitic topologies, with the metal center replacing the tetrahedral silicon atom and the linker replacing the oxygen 

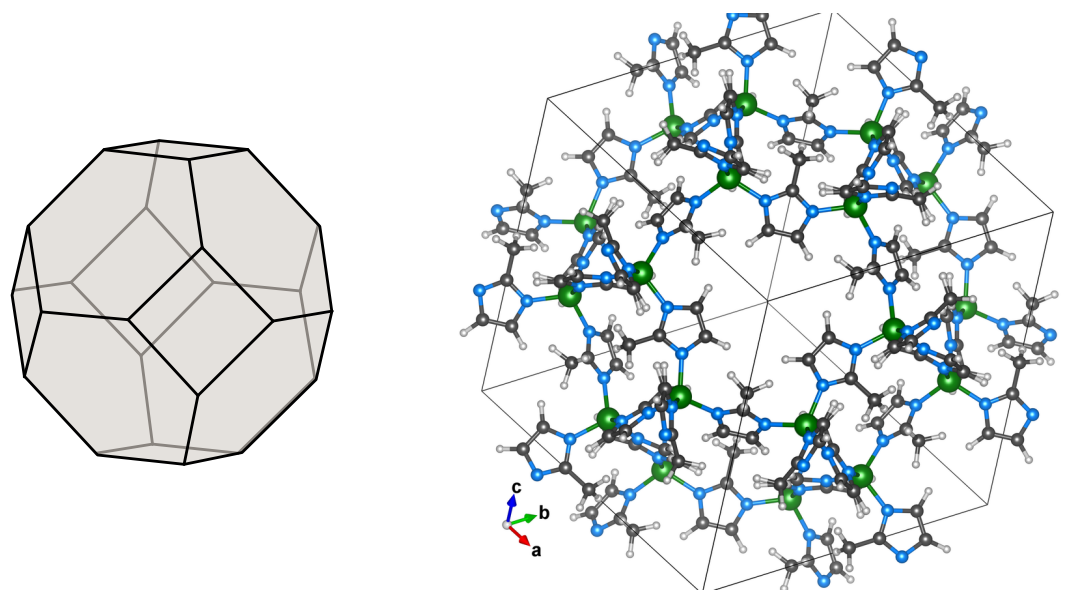

Figure 1: (left) schematic representation of the sodalite topology. (right) molecular structure of ZIF-8. The color code is black for carbon, white for hydrogen, blue for nitrogen and green for zinc. 4-members and 6-members windows are delimited by zinc atoms.

atoms in the $\mathrm{SiO}_{2}$ building block. ZIF-8 has formula $\mathrm{Zn}(\mathrm{mim})_{2}$ and adopts the sodalite (sod) topology. In this topology, large quasi-spherical pores corresponding to the sodalite cages are connected by windows formed by 6 and 4 zinc atoms (see Figure 1). In ZIF-8, the 4 members windows are too small for any molecules to go through, and all of the connectivity of the pore space happens though the 6 members windows. ZIF-8 is hydrophobic, ${ }^{17}$ and presents interesting behavior upon intrusion. While osmotic pressure effects do not depend on the chemical nature of the ions, ZIF-8 shows changes from one energetic behavior to another when the ion nature changes while keeping concentration constant. 18 ZIF-8 behavior can also be tuned chemically, by modifying the nature of the linkers, for example changing the 2-methylimidazolate to a 2-chloroimidazolate increases the intrusion pressure. 29

However, the exact mechanism and behavior at the molecular level upon intrusion of electrolytes in ZIF-8 are still unknown. In this work, we used classical molecular simulations to study the structure, dynamics and energetic implications of confining water and aqueous solutions of $\mathrm{LiCl}$ in ZIF-8. Using these simulations, we were able to explore different aspects of the $\{$ water, ZIF-8 3 and $\{$ electrolyte, ZIF-8 systems. We describe below the structure of the liquids and the influence of confinement, their dynamics, the mechanical properties of ZIF-8 and the impact of liquid intrusion on them. We conclude by a series of considerations 
on the energetics of intrusion, and results on the thermodynamics and the free energy of entry of ions in ZIF-8.

\section{Computational methods}

We ran classical molecular dynamics (MD) simulations and umbrella sampling simulations using the LAMMPS ${ }^{30}$ code. The umbrella sampling simulations additionally used the colvars $^{31}$ module for collective variables. We used a combination of different force fields for the component of the system: a rigid $\mathrm{SPC} / \mathrm{E}^{32}$ for water, for its ability to describe the dynamics of liquid water and the solvation of ions; a flexible force-field adapted from AMBER by Zheng et al. ${ }^{33}$ for the description of the ZIF-8 framework; and a combination of electrostatic and Lennard-Jones potentials for the ions, reproduced in supplementary information Table $\left[1, \frac{34}{34}\right.$ We used Lorentz-Berthelot mixing rules for cross-terms in LennardJones potential, and Ewald summation to account efficiently for electrostatic interactions. We used a cutoff of $8.5 \AA$ for both the Lennard-Jones potential and the separation between real space and Fourier space in the Ewald summation.

After an initial energy minimization, we carried all simulations in the isothermal-isobaric $(N, P, T)$ ensemble with a timestep of $1 \mathrm{fs}$, using a Nosé-Hoover thermostat with a time constant of 1 ps and a Nosé-Hoover barostat with a time constant of 10 ps. We allowed the barostat to make arbitrary changes to unit cell lengths and tilt factors (fully flexible anisotropic

cell), while imposing an isotropic pressure to the system. Unless specified otherwise, we ran all simulations in the $(N, P, T)$ ensemble for $10 \mathrm{~ns}$, and only used the last $4 \mathrm{~ns}$ for analysis.

We used three different types of systems in this study. First, bulk liquids at different $\mathrm{LiCl}$ concentration: $0 \mathrm{~mol} / \mathrm{L}$ (pure water), $1 \mathrm{~mol} / \mathrm{L}, 5 \mathrm{~mol} / \mathrm{L}, 10 \mathrm{~mol} / \mathrm{L}, 15 \mathrm{~mol} / \mathrm{L}$ and $20 \mathrm{~mol} / \mathrm{L}-$ the experimental solubility of $\mathrm{LiCl}$ in water at $25{ }^{\circ} \mathrm{C}$ is $19.87 \mathrm{~mol} / \mathrm{L}$. Then we simulated a $3 \times 3 \times 3$ supercell of ZIF-8, with liquid confined inside the pores, at the same concentrations as the bulk liquid. Finally, we used a $2 \times 2 \times 3$ ZIF- 8 supercell containing pure water together 
with a $34 \AA$ cubic reservoir of water on top (this system featuring an explicit ZIF-8/liquid interface) for the umbrella sampling simulations.

We generated the initial configuration using the packmol software, ${ }^{35}$ randomly placing the desired number of particles in the system. We started with bulk electrolyte in a $32 \AA$ cubic box containing 750 water molecules for the pure water, and added ions for the different $\mathrm{LiCl}$ concentrations. For the liquids confined in ZIF-8, we needed to know how many molecules would fit in the ZIF-8 pores. For that, we ran a constant pressure simulation with a reservoir of pure water outside an empty $2 \times 2 \times 3$ ZIF- 8 supercell at $0.5 \mathrm{GPa}$. We ran the simulation for $10 \mathrm{~ns}$ and counted 75 water molecules in one unit cell. We then ran simulations of the bulk liquid at varying concentration at the constant pressure of $0 \mathrm{GPa}$, and recorded the corresponding particles density. Using the density of pure water, we mapped the 75 molecules per unit cell to an accessible porous volume of $2.286 \mathrm{~nm}^{3}$, or $46 \%$ of the unit cell. From this volume and the density of the bulk liquids, we could now compute the number of molecule to put in the $3 \times 3 \times 3$ supercell for each concentration. The resulting system was a cubic box of $51 \AA$, containing roughly 13000 atoms.

For analysis, we used the chemfiles library and its cfiles command-line interface (available online at https://github.com/chemfiles/cfiles). Representative input files for the simulations are available online in the data repository at https://github.com/fxcoudert/ citable-data.

\section{Results and discussion}

\subsection{Structure of the liquid}

From the point of view of the liquid, the principal effect of intrusion is the confinement of the fluid to a pore space of nanometric dimensions, i.e. the nanoporous material acts as a host matrix - although a flexible one. Here, we look at the effects of this confinement on the liquid structure, as a function of the electrolyte concentration. In order to characterize 
the structure of the liquid and the solvation of ions confined in ZIF-8, we computed radial distribution function for each pair of atom types in the system. They are presented in supplementary information Figures S1 and $\mathrm{S2}$. Integrating the radial distribution function until the first minimum gives the number of neighbors in the first solvation shell of each atom. The evolution of this number of neighbors as a function of $\mathrm{LiCl}$ concentration is presented in Figure 2, for both the bulk liquid and the confined liquid.

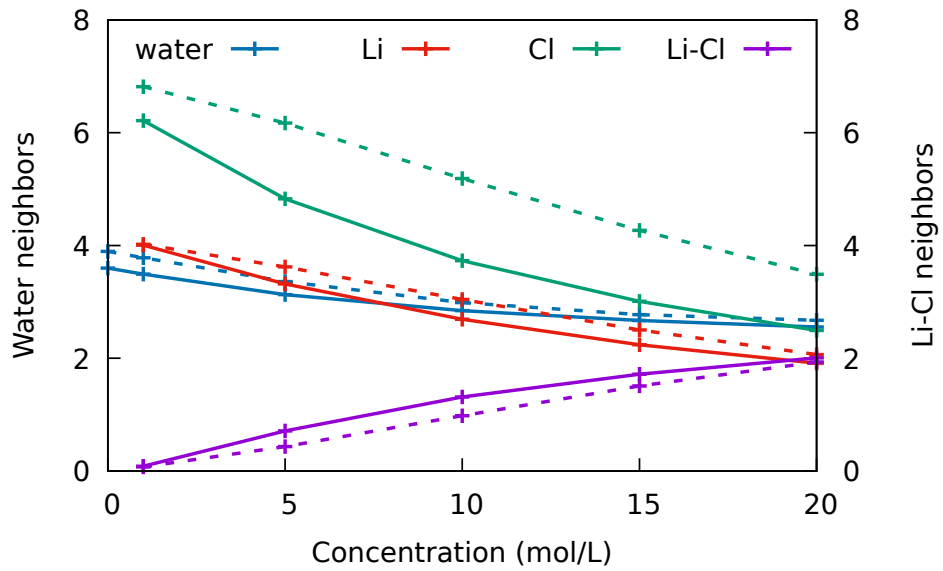

Figure 2: Number of water neighbors in the first solvation shell in the confined liquid (plain lines) and the bulk liquid (dotted lines) as function of the concentration, at the constant pressure of $0 \mathrm{GPa}$. The number of chlorine neighbors for lithium ions is also represented.

The first thing we can see here is that, for all species ( $\mathrm{Li}, \mathrm{Cl}$ and water), the number of neighboring water molecules - i.e., the solvation - decreases as the $\mathrm{LiCl}$ concentration increases. At $0 \mathrm{~mol} / \mathrm{L}$ and $1 \mathrm{~mol} / \mathrm{L}$, there are more than enough water molecules with respect to the ions (roughly 55 water molecule per ion at $1 \mathrm{~mol} / \mathrm{L}$ ) so that they can be in their ideal solvation state: $4 \mathrm{H}_{2} \mathrm{O}$ per $\mathrm{Li}^{+}$, and $6 \mathrm{H}_{2} \mathrm{O}$ per $\mathrm{Cl}^{-}$. But as the concentration increases, there are fewer available water molecules and the ions have to accommodate by having less molecule in their first solvation shell. At $20 \mathrm{~mol} / \mathrm{L}$, there are only 2.7 water molecule available for each lithium ion; and each cation is thus surrounded by 1.9 water molecule, less than half its complete solvation state. The same is true for water/water coordination through hydrogen bonds, as the water molecules compete with the ions to surround themselves with other water molecules. 
In the bulk liquid, the loss of neighbors is linear with the concentration, as all the molecules in the system are able to adapt to find the state of largest possible solvation. In the confined liquid however, the molecules are geometrically constrained by the presence of the ZIF-8 framework, which manifests as an excluded volume, and thus are not able to adapt as well to the increase in $\mathrm{LiCl}$ concentration, making the number of neighbors drop faster. We also note a slight increase in the number of chlorine neighbors of lithium ions at intermediate concentration, compared to the bulk: the effect of confinement, by diminishing the solvation of the ions, favors the occurrence of anion-cation pairs. The number of lithium neighbors for chlorine ions is the same as the number of chlorine neighbors for lithium ions.

Going from bulk liquid to confined liquid also changes the number of neighbors for water and $\mathrm{Cl}$ ions, even at $1 \mathrm{~mol} / \mathrm{L}$. In addition to preventing a full reorganization of the water molecules when the concentration increases, the presence of the framework also affects the structure of the solvation shells. Molecules close to the framework can only have neighbors from the liquid on one side — an excluded volume effect. However, the framework also has an effect at longer range, the available space in the pores dictating the arrangement of molecules. Instead of being widely distributed, the molecules are restricted to specific preferential locations, due to host-guest interactions. This effect is particularly visible on Figure 3, and is stronger on $\mathrm{Cl}$ /water pairs than water/water or $\mathrm{Li} /$ water, as the $\mathrm{Cl}$ has a larger radius and binds to the hydrogen atoms in water, making its solvation sphere both bigger and "softer".

We also present in Figure 3 the density profile of atoms in the confined liquid, represented in the $x y$ plane, averaged over $z$ and the $3 \times 3 \times 3$ supercell. To account for cell deformation during the $(N, P, T)$ simulations, we used fractional coordinates to represent the positions of atoms.

Here we clearly see the long distance structuration of water inside the ZIF-8 pores. At low concentration $(1 \mathrm{~mol} / \mathrm{L})$, the water molecules occupy very well-defined sites, in particular inside the windows between two neighboring cages. As the concentration increases, this 

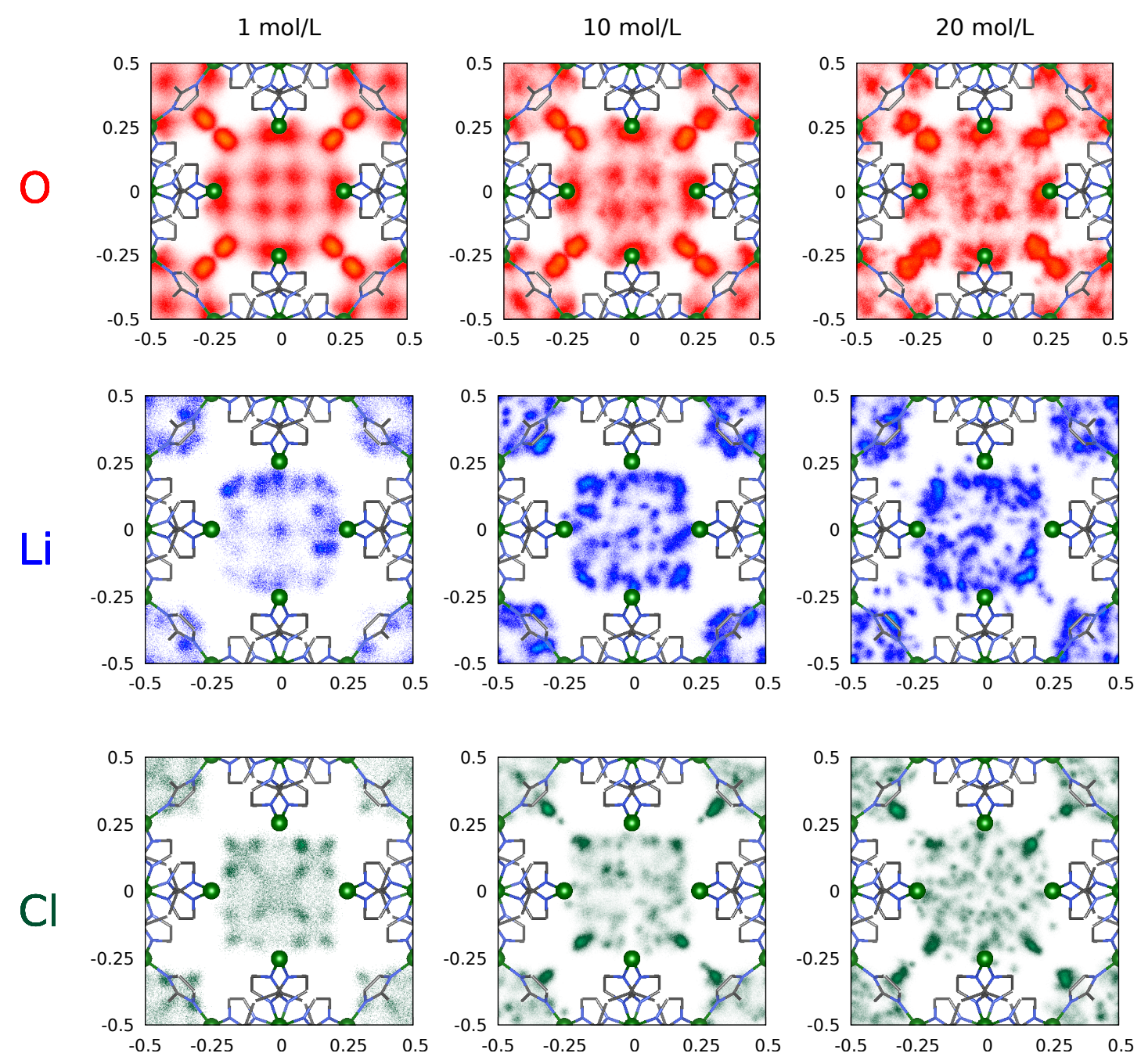

Figure 3: Two-dimensional density profile of oxygen (top), lithium (middle) and chlorine (bottom) atoms in $\mathrm{LiCl}$ electrolyte confined in $\mathrm{ZIF}-8$ at $0 \mathrm{GPa}$ as a function of $\mathrm{LiCl}$ concentration (left to right). The atoms from the ZIF-8 framework are superimposed, with some linkers omitted for clarity between the four central zinc atoms.

organization is perturbed by the ions inserted in the water molecules' network - however this effect is relatively small, and the water distribution is not greatly affected. The same can be observed in the distribution of chlorine ions, with a well defined, high symmetry distribution at low concentration, but as the concentration increases the distribution of ions becomes more and more distributed over the whole pore space. Concerning lithium ions, they present a looser arrangement inside the pores, and are distributed relatively evenly, yet present a preferential occupation next to the water molecules in the 6-member windows (in 
the diagonal in Figure 3).

These results can be explained by both the difference in kinetic radius ${ }^{36}$ for water molecules (2.65 $\AA$ ), chlorine ions $(3.2 \AA)$ and lithium ions $(2.1 \AA)$, as well as the strong attraction between water oxygen atoms and lithium (see the radial distribution functions in Figure S2). The difference in size allows lithium ions to fit in smaller spaces, and an even distribution of ions will increase the total entropy of the system. At the same time, chlorine ions and water molecules are more polarizables than lithium, and as such will have stronger interactions with the aromatic linkers, making it preferable for them to take the highly organized arrangement we observe.

\subsection{Dynamics under confinement}

We used two different indicators to quantify the dynamics of water molecules confined in ZIF-8 and the impact of $\mathrm{LiCl}$ concentration and pressure on these dynamics. The first one is based on the lifetime of hydrogen bonds between water molecules. Following Luzar and Chandler, ${ }^{37}$ we characterize the presence of a hydrogen bond between two water molecules

as two oxygen atoms separated by less than $3.5 \AA$, with an oxygen-oxygen-hydrogen $(\widehat{\mathrm{OOH}})$ angle less than $30^{\circ}$. We then computed the time autocorrelation function of the hydrogen bond existence functions $H(t)$ - set to 1 if the bond exists at time $t, 0$ if is does not exists as:

$$
C_{\mathrm{hbonds}}(t)=\left\langle H\left(t_{0}\right) \cdot H\left(t_{0}+t\right)\right\rangle_{t_{0}}
$$

The decay of this autocorrelation function, presented in Figure 4, is characteristic of the dynamics of the hydrogen bond network and the lifetime of individual hydrogen bonds.

This geometric definition of hydrogen bonds has a minor inconvenient: small fluctuations of the atomic positions, near the cut-off values, can be mistaken for hydrogen bond forming and breaking. To overcome this issue, we also computed the time autocorrelation of the 
orientation vector $\boldsymbol{u}(t)$ of water molecules:

$$
C_{\mathrm{rot}}(t)=\left\langle P_{2}\left(\boldsymbol{u}\left(t_{0}\right) \cdot \boldsymbol{u}\left(t_{0}+t\right)\right)\right\rangle_{t_{0}},
$$

where $P_{2}(x)$ is the second order Legendre polynomial $P_{2}(x)=\frac{1}{2}\left(3 x^{2}-1\right) . \frac{38}{\text { The results }}$ for rotational correlation are presented in supporting information Figure S9 and Table S3. Because water is a strongly associated liquid, breaking a hydrogen bond is predominantly correlated to rotational jumps, both autocorrelations behave in very similar ways, and we focus here the discussion on hydrogen bonds decays.
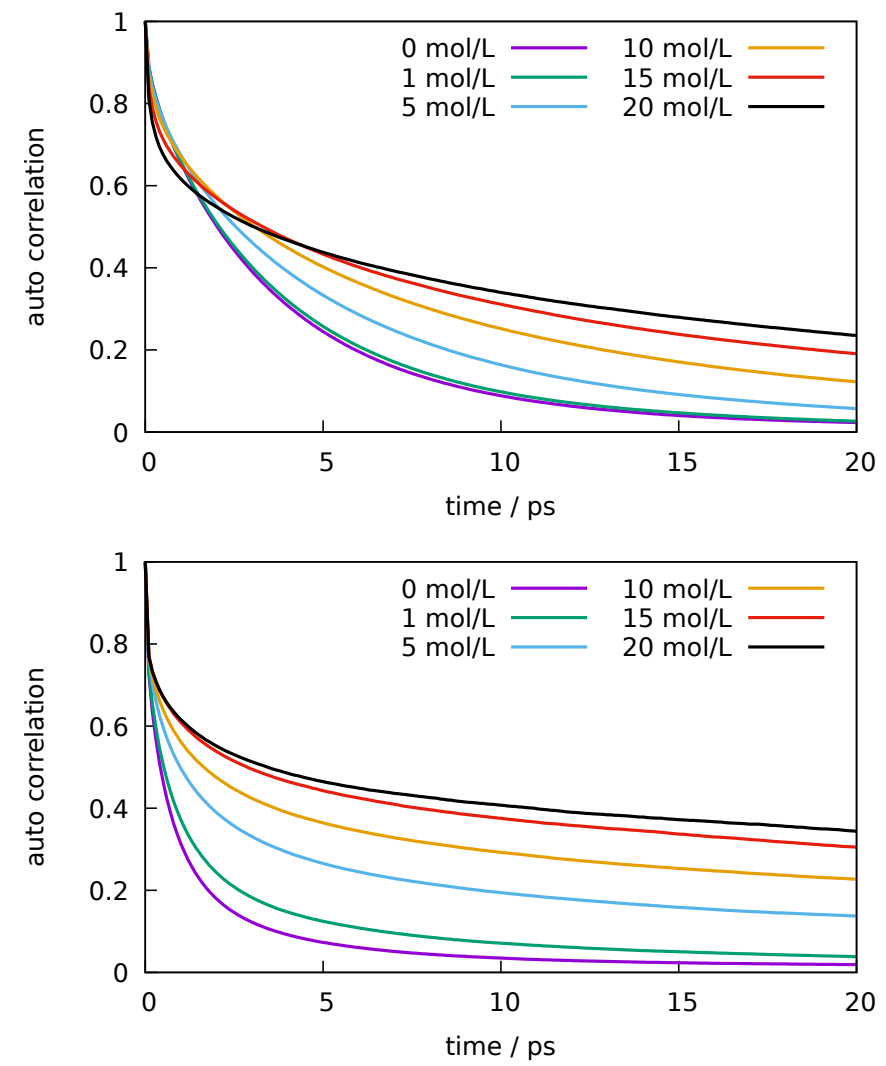

Figure 4: Hydrogen bonds existence autocorrelation in bulk (top) and confined (bottom) electrolyte as function of $\mathrm{LiCl}$ concentration.

We then fitted all the autocorrelation functions with bi-exponential functions:

$$
f(t)=A_{1} e^{-t / \tau_{1}}+A_{2} e^{-t / \tau_{2}},
$$


where $\tau_{1}$ and $\tau_{2}$ are the two time scales of decay, and $A_{1}$ and $A_{2}$ are their relative weights. The resulting fit parameters are presented in supporting information Table $\mathrm{S} 2$, The corresponding data is presented in Figure 5 at the pressure of $0 \mathrm{GPa}$. We found that the pressure only have a negligible influence on both the lifetimes and the weights of these lifetimes. Similar curves for all pressures are presented in supplementary information Figures S7 and S8.
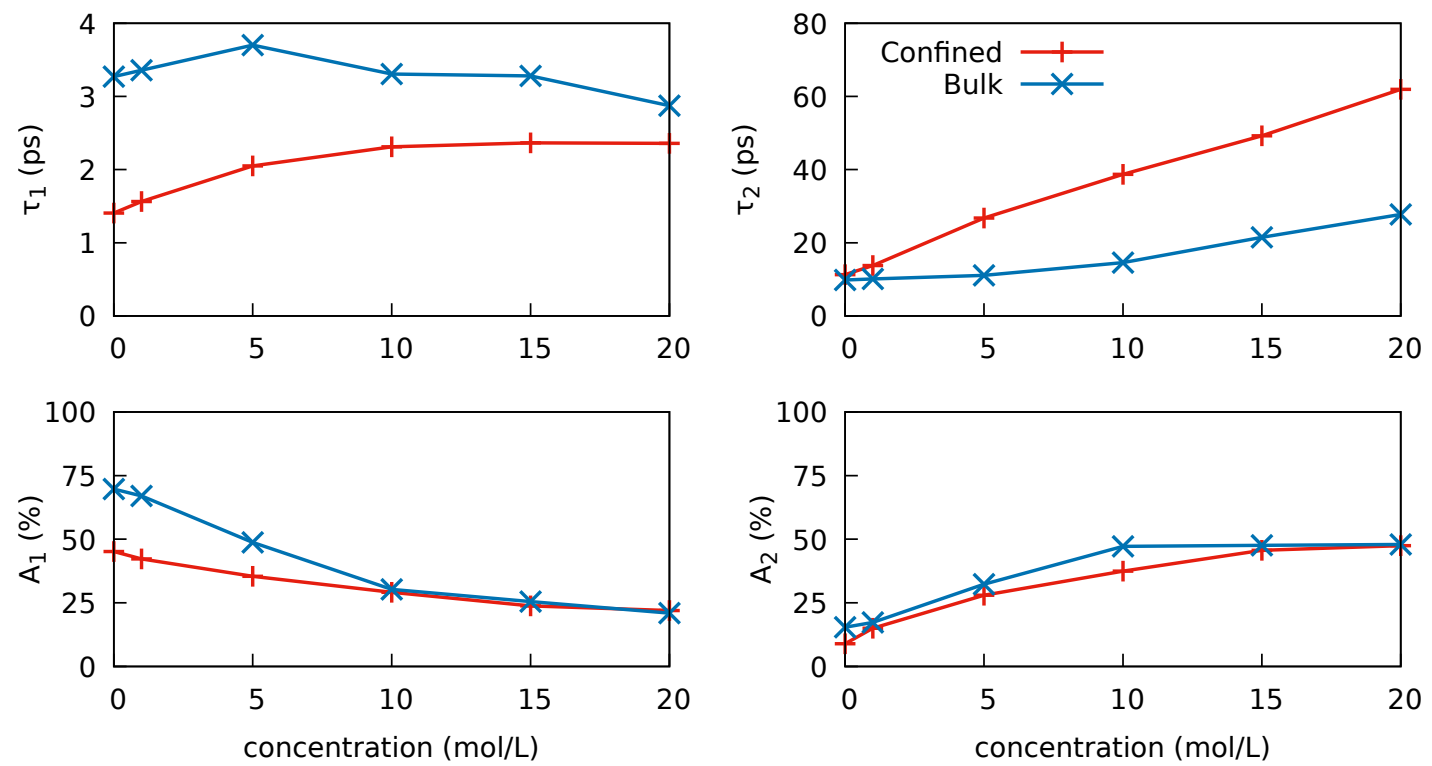

Figure 5: Variations of the time constant and weights of the bi-exponential hydrogen bonds autocorrelation decay at $0 \mathrm{GPa}$ in bulk and confined liquid as function of the $\mathrm{LiCl}$ concentration.

In the bulk liquid, the shortest lifetime is almost constant as the concentration increases, but the weight of this fast process $\left(A_{1}\right)$ decreases. This suggests that this fastest lifetime is associated with hydrogen bonds between water molecules surrounded only by other water molecules. As more and more ions are added in the system, water molecules are less likely to be surrounded only by other water molecules — as is indicated by the results we presented in section 3.1. We also note that this lifetime is smaller in the confined liquid than it is in the bulk phase, which has already been shown for water at interfaces: Fogarty et al. ${ }^{38}$ showed that it is linked to the librational motions of the $\mathrm{OH}$ bonds, and the dynamics of these "dangling" OH groups at interfaces is faster than bulk. ${ }^{39}$ On the other hand, the second lifetime, associated to the slowest process, increases with concentration, as well as the 
corresponding weight $\left(A_{2}\right)$. This points to hydrogen bonds between water molecules bounded to ions.

In the confined liquid, weights still evolve in the same way with respect to the concentration, which point to them being associated with the same kind of hydrogen bonds. The second lifetime increases in the confined liquid compared to the bulk liquid. This slowdown of water dynamics under confinement is well-known, $\underline{38}$ and has been observed in many classes of nanoporous materials. $\frac{26 / 40}{42}$ It is attributed to the stronger organization of water as well as water molecules finding fewer partners for hydrogen bond exchange. The same arguments also apply to the increase in $\mathrm{LiCl}$ concentration, both in the bulk and confined liquid. This is coherent with what we see on the changes on bulk modulus as concentration changes in Table 1 .

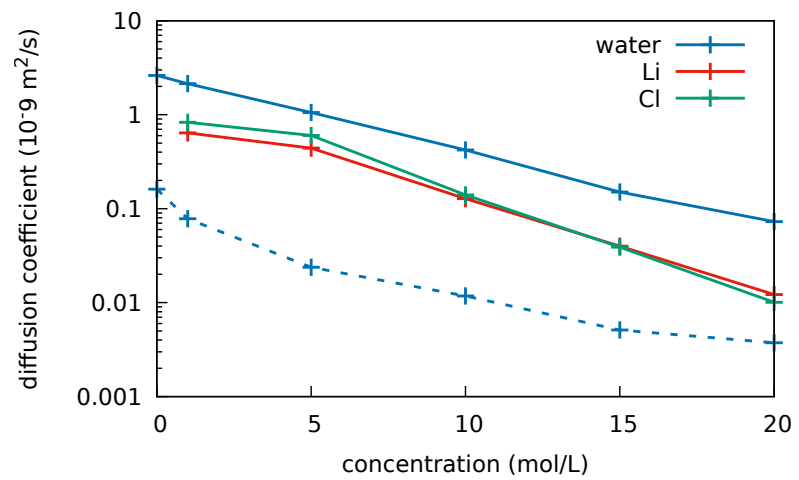

Figure 6: Diffusion coefficients from mean square distance in bulk liquid (plain lines) and confined liquid (dashed line). No diffusion coefficient was extracted for $\mathrm{Li}$ and $\mathrm{Cl}$ in confined liquid, since they don't diffuse through ZIF-8 windows.

A separate way to quantify the dynamics of the confined and bulk liquid is to look at the diffusion of different species in the liquids. The diffusion coefficient $D$ can be extracted from the mean square displacement using the Einstein relation:

$$
\left\langle\left(\vec{r}\left(t-t_{0}\right)-\vec{r}\left(t_{0}\right)\right)^{2}\right\rangle_{t_{0}}=6 D t
$$

This relation is only valid for long times, and if diffusion indeed occurs in the system. We computed the mean square displacement for water, lithium and chlorine in both the bulk 
and confined liquid, the corresponding curves are presented in supplementary information Figures $\mathrm{S13}$ and $\mathrm{S14}$. We then extracted the coefficient diffusion from these curves when possible, they are shown as a function of concentration in Figure6. We could not get diffusion coefficients for the ions in the confined liquid, since they don't cross the windows — as explained in section 3.6 .

The diffusion coefficient for pure water $\left(2.6 \times 10^{-9} \mathrm{~m}^{2} / \mathrm{s}\right)$ matches previous studies of the same water model.$\stackrel{43}{[3}$ In the bulk liquid, as the ion concentration increases, both the self diffusion of water and the diffusion of the ions slows down dramatically. At $20 \mathrm{~mol} / \mathrm{L}$, the diffusion coefficient of water is only $0.072 \times 10^{-9} \mathrm{~m}^{2} / \mathrm{s}$, and the coefficient of the ions is $0.012 \times 10^{-9} \mathrm{~m}^{2} / \mathrm{s}$. Concerning the confined liquid, we observe the same slow down of the dynamics as usual, the water being one order of magnitude slower when confined compared to the bulk. Then, concentration effects are added, slowing the diffusion even further. Diffusion thus shows the same behavior as the hydrogen bond dynamics, with two independent and additive processes (confinement and ion presence) explaining the global slowdown of the dynamics.

\subsection{Deformation of the framework}

We have studied the property of the confined liquid inside the ZIF-8 framework, and the changes in the structure and dynamics of this liquid as it goes from a bulk to a confined state. It is also interesting to look at the changes the framework undergoes when going from an empty state to an intruded state. Macroscopic changes to the volume are relatively small (less than 4\%), and presented in the next sections. In this section we will look at the internal deformations of the framework under intrusion.

Following previous works on the flexibility of ZIF-8, $\underline{44}+46$ we used the distribution of the $\mathrm{Zn}-\mathrm{Zn}-\mathrm{Zn}-\mathrm{CH}_{3}$ dihedral angle (also called swing angle) to characterize the deformations of the framework under intrusion. This angle describe the rotation of the methyl-imidazolate linker around the surrounding zincs axis. The distributions for empty and intruded ZIF-8 
are presented in Figure 7.

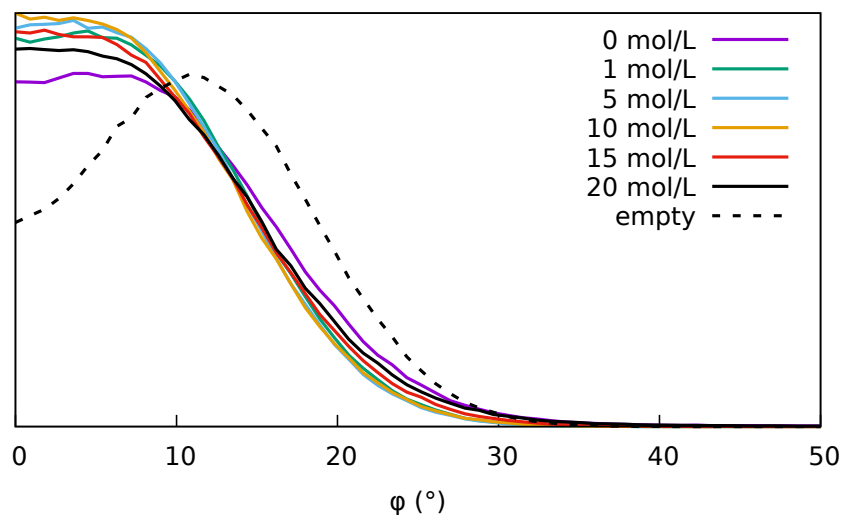

Figure 7: Swing dihedral angle distribution in empty and intruded ZIF-8 at all the LiCl concentrations.

The distribution for the empty framework reproduces the already published results: it is a Gaussian distribution, centered around the equilibrium value of $15^{\circ}$, and with fluctuations of the order of $10^{\circ}$. The distributions for ZIF-8 containing the electrolyte liquid does not seems to depend on the electrolyte concentration. Instead they are all centered around $5^{\circ}$, and still have the same order of magnitude for fluctuations. This is to be contrasted with the effect of nitrogen adsorption at $77 \mathrm{~K}$ in ZIF-8; $\frac{44}{4}$ where the equilibrium value goes from $10^{\circ}$ to $25^{\circ}$ as the pores fill up with nitrogen. It is however interesting to note that the presence of ions does not impact the structure more than the presence of pure water does. This is coherent with the results presented in Figure 3, where the water molecules are located inside the 6-member windows. Water molecules take the place of linkers that rotate to accommodate them in the windows. As ions never enter this window, they don't have any effect on the linkers rotation.

\subsection{Elastic properties}

Given its potential applications in mechanical energy storage, one of the most important properties of the ZIF-8/electrolyte system is its mechanical behavior, and notably its stability under high pressure. As an increasing stress is applied to a material, it will first deform in a reversible and linear fashion, in what is called the linear elastic regime. Under higher stress, 
the material will start to deform in an irreversible way, and finally break. Information on the linear elastic regime and its extent is useful to study the stability of materials under stress, as stiffer materials are generally tougher and able to bear higher stress before failing. This is particularly important for soft nanoporous materials, where the mechanical stability range is lower than in inorganic porous materials such as zeolites, and where pressure-induced amorphization is common at moderate (sub-gigapascal) pressures. 47,49

We probed the mechanical response of the ZIF-8/electrolyte system using direct simulations of the system under explicit hydrostatic stress. We first note that even though we allowed arbitrary changes to the unit cell lengths and tilt factors, all the simulations cells remained orthorhombic. The Figure 8 present the changes in volume as the pressure increases, for all the liquid concentrations. We can see that up to $1 \mathrm{GPa}$, the deformation remains in the elastic regime, and that the response is almost linear. Moreover, confining an electrolyte in the ZIF-8 structure do not drastically affect the mechanical properties of the system.

From these curves, we extracted the bulk modulus $K$ of the system, defined as

$$
K=-V\left(\frac{\partial P}{\partial V}\right)_{N, T}
$$

We evaluated the derivative $(\partial P / \partial V)$ in the above formula as the slope of the curves from $0 \mathrm{GPa}$ to $1 \mathrm{GPa}$. The values of the bulk modulus in various conditions are reported in Table 1 . The value for the empty ZIF-8 is close to the experimental ${ }^{50}$ value of $7.8 \mathrm{GPa}$. The bulk moduli of pure water and $1 \mathrm{~mol} / \mathrm{L}$ are further away from the experimental values ${ }^{51}$ of $2.4 \mathrm{GPa}$ and 2.6 GPa respectively. These differences are likely coming from the force-fields we used, which were not parameterized on mechanical properties of the framework or the liquids.

The trends, however, are interesting to see. Adding water to the pores of ZIF-8 only changes the bulk modulus by a moderate amount (10\%), meaning that most of the stiffness comes from the ZIF-8 framework - the stiffer component of the two. However, adding ions to the liquid has a larger effect, both in the bulk state and the confined state, with the 

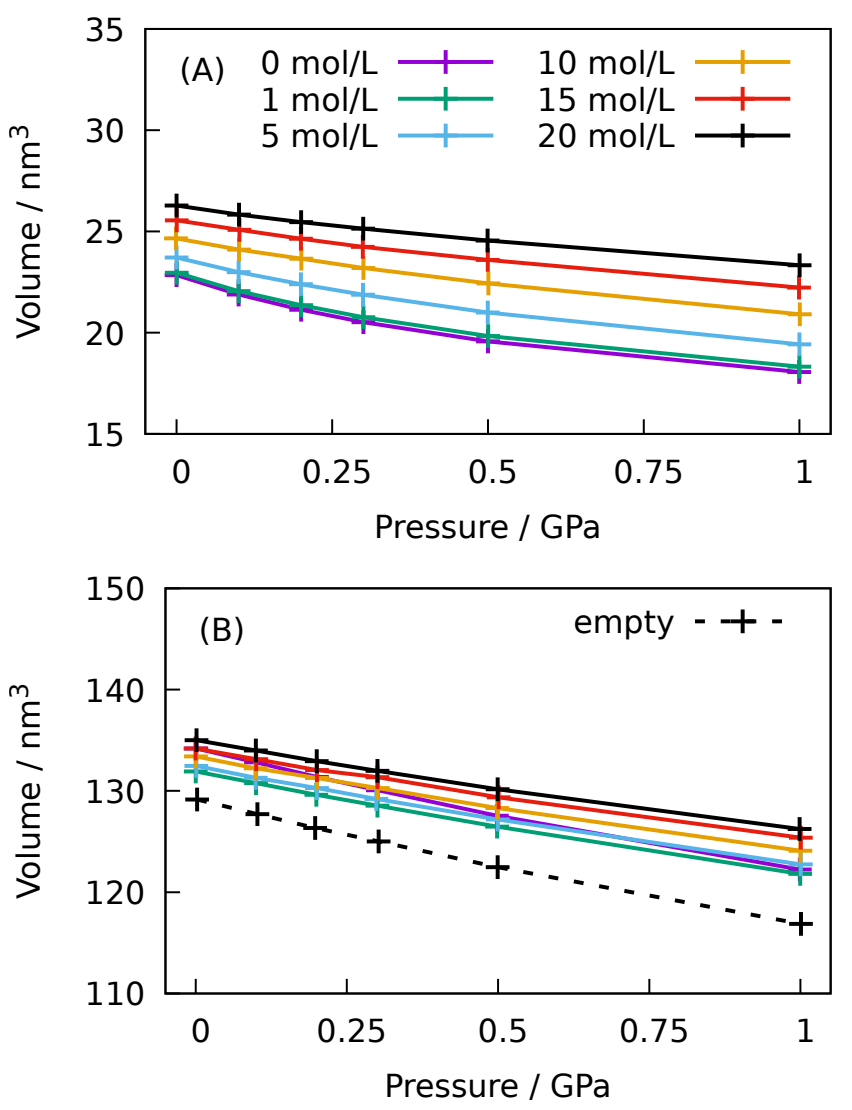

Figure 8: Deformation of bulk liquid (A) and ZIF-8 with confined electrolyte (B) as function of the pressure. Lines connect the different simulations using the same concentration.

bulk modulus increasing by up to $50 \%$ at $20 \mathrm{~mol} / \mathrm{L}$ with respect to the empty framework. Since we generated the structures in such a way that the volume occupied by the liquid is always the same, the increase in bulk modulus is not be related to changes in the size occupied by the ions relatively to water. Rather, this increase in bulk modulus comes from the stronger interactions between ions and water molecules, compared to interactions between water molecules. The interactions between lithium cations and water molecules are stronger than water-water hydrogen bonds, and will make the liquid less compressible, especially at high concentration where, statistically, all water molecules are bonded to at least one lithium atom. This is further supported by the fact that the bulk modulus increases by the same order of magnitude $(\approx 5 \mathrm{GPa})$ in both the bulk liquid and the confined liquid in ZIF-8. 
Table 1: Bulk modulus of the bulk electrolyte liquids and of ZIF-8 containing a confined electrolyte liquid.

\begin{tabular}{ccc}
\hline Concentration & Bulk liquid & Liquid $\in$ ZIF-8 \\
\hline $0 \mathrm{~mol} / \mathrm{L}$ & $4.3 \mathrm{GPa}$ & $11.2 \mathrm{GPa}$ \\
$1 \mathrm{~mol} / \mathrm{L}$ & $4.4 \mathrm{GPa}$ & $13.0 \mathrm{GPa}$ \\
$5 \mathrm{~mol} / \mathrm{L}$ & $5.0 \mathrm{GPa}$ & $13.6 \mathrm{GPa}$ \\
$10 \mathrm{~mol} / \mathrm{L}$ & $6.1 \mathrm{GPa}$ & $14.4 \mathrm{GPa}$ \\
$15 \mathrm{~mol} / \mathrm{L}$ & $7.2 \mathrm{GPa}$ & $15.2 \mathrm{GPa}$ \\
$20 \mathrm{~mol} / \mathrm{L}$ & $8.4 \mathrm{GPa}$ & $15.4 \mathrm{GPa}$ \\
\hline Empty ZIF-8 & & $10.5 \mathrm{GPa}$ \\
\hline
\end{tabular}

\subsection{Thermodynamics of the intrusion}

In order to shed light into the energetics and thermodynamics of electrolyte intrusion in ZIF-8, we extracted the potential energy for various sub-components of the total system by taking the average value of the interaction energy of the corresponding sub-components. The resulting average energies are presented in Table 2; where $E_{\text {total }}$ is the total potential energy of the electrolyte confined in ZIF-8; $E_{\mathrm{ZIF}}$ is the interaction of ZIF-8 with itself; $E_{\mathrm{LiCl}}$ is the interaction of the confined electrolyte with itself; and $E_{\mathrm{ZIF} / \mathrm{LiCl}}$ is the interaction of the electrolyte with the ZIF-8. $E_{\mathrm{LiCl}}^{\mathrm{bulk}}$ refers to the total potential energy of the bulk electrolyte with the same number of particles as the confined one. Every quantity is expressed for one unit cell of ZIF-8, plus the confined liquid inside. From these values, we can extract a few thermodynamic quantities of interest, presented in Table 3 ;

$$
\begin{gathered}
\Delta E_{\mathrm{ZIF}}(c)=E_{\mathrm{ZIF}}(c)-E_{\mathrm{ZIF}}^{\mathrm{empty}} ; \\
\Delta E_{\mathrm{LiCl}}(c)=E_{\mathrm{LiCl}}(c)-E_{\mathrm{LiCl}}^{\mathrm{bulk}}(c) ; \\
\Delta H_{\mathrm{intr}}(c)=E_{\mathrm{total}}(c)-E_{\mathrm{LiCl}}^{\text {bulk }}(c)-E_{\mathrm{ZIF}}^{\mathrm{empty}} .
\end{gathered}
$$


Table 2: Average interaction energy in $\mathrm{kcal} / \mathrm{mol}$ per unit cell for various subsystems. See the text for the definition of each sub-system. For reference, $k_{B} T$ is $0.6 \mathrm{kcal} / \mathrm{mol}$ at $300 \mathrm{~K}$.

\begin{tabular}{cccccc}
\hline LiCl & $E_{\text {Total }}$ & $E_{\mathrm{ZIF}}$ & $E_{\mathrm{LiCl}}$ & $E_{\mathrm{LiCl} / \mathrm{ZIF}}$ & $E_{\mathrm{LiCl}}^{\text {bulk }}$ \\
\hline Empty & -746.8 & -746.8 & & & \\
$0 \mathrm{~mol} / \mathrm{L}$ & -1617 & -758.1 & -703.1 & -155.5 & -822.9 \\
$1 \mathrm{~mol} / \mathrm{L}$ & -1859 & -739.5 & -974.0 & -145.8 & -1080 \\
$5 \mathrm{~mol} / \mathrm{L}$ & -2753 & -738.3 & -1864 & -149.7 & -1995 \\
$10 \mathrm{~mol} / \mathrm{L}$ & -3628 & -735.5 & -2744 & -148.6 & -2898 \\
$15 \mathrm{~mol} / \mathrm{L}$ & -4295 & -732.5 & -3416 & -146.0 & -3581 \\
$20 \mathrm{~mol} / \mathrm{L}$ & -4818 & -728.4 & -3949 & -140.9 & -4117 \\
\hline
\end{tabular}

$\Delta E_{\mathrm{ZIF}}$ is the energetic change in ZIF-8 during intrusion; $\Delta E_{\mathrm{LiCl}}$ is the energetic change in the electrolyte during intrusion; and $\Delta H_{\text {intr }}$ is the intrusion enthalpy, i.e. the enthalpy change during the ZIF-8 + liquid $\rightarrow$ liquid $\in$ ZIF-8 process. The sign convention is taken so that all of these energies are negative when the confined state is more stable.

Table 3: Derived thermodynamic quantities in kcal/mol per unit cell. See the text for the definition of each quantity.

\begin{tabular}{c|ccc}
\hline LiCl & $\Delta E_{\text {ZIF }}$ & $\Delta E_{\mathrm{LiCl}}$ & $\Delta H_{\text {intr }}$ \\
\hline $0 \mathrm{~mol} / \mathrm{L}$ & -11.3 & -120 & -47.3 \\
$1 \mathrm{~mol} / \mathrm{L}$ & 7.3 & -106 & -32.2 \\
$5 \mathrm{~mol} / \mathrm{L}$ & 8.5 & -131 & -11.2 \\
$10 \mathrm{~mol} / \mathrm{L}$ & 11.3 & -154 & 16.8 \\
$15 \mathrm{~mol} / \mathrm{L}$ & 14.3 & -165 & 32.8 \\
$20 \mathrm{~mol} / \mathrm{L}$ & 18.4 & -168 & 45.8 \\
\hline
\end{tabular}

We can see in Table 3 that the intrusion process has a relatively small impact on the ZIF: the energy difference between the empty and intruded states $\Delta E_{\mathrm{ZIF}}$ is in the range of tens of $k T$ (at $300 \mathrm{~K}, k T \approx 0.6 \mathrm{kcal} / \mathrm{mol}$ ). The ZIF-8 framework is slightly destabilized (energetically) in presence of the intruded liquid except at $0 \mathrm{~mol} / \mathrm{L}$, where it is slightly stabilized. The overall effect is small compared to the two next trends we see. We have already shown that 
the presence of ions have little to no effect on the ZIF-8 structure in section 3.3 .

First, the liquid is always more energetically stable in the intruded phase than in the bulk phase $\left(\Delta E_{\mathrm{LiCl}}\right)$. This might seems strange as ZIF-8 is an hydrophobic material, but the values presented only account for energetic contributions, and do not contain entropy. Figure 3 shows that the entropy of the confined liquid can indeed be expected to be lower than the entropy of the bulk liquid, because of the strong organization of the confined fluid. As the $\mathrm{LiCl}$ concentration increase, the intruded phases becomes more and more stabilized, the ions adding additional rigidity and strong interactions in the pores network.

Secondly, we see that the energetic behavior of the whole process $\left(\Delta H_{\text {intr }}\right)$ is more complex: the intrusion process is energetically favorable for low concentrations $(\leq 5 \mathrm{~mol} / \mathrm{L})$, and becomes unfavorable at higher $\mathrm{LiCl}$ concentrations $(\geq 10 \mathrm{~mol} / \mathrm{L})$. The interaction between the liquid and ZIF-8 $\left(E_{\mathrm{LiCl} / \mathrm{ZIF}}\right.$ in Table 2$)$ makes for the difference between $\Delta E_{\mathrm{ZIF}}+\Delta E_{\mathrm{LiCl}}$

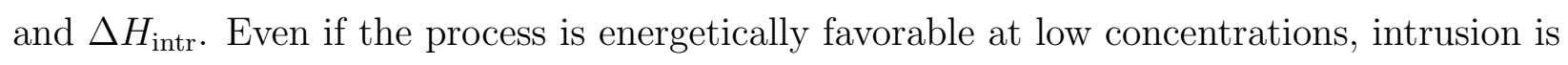
not spontaneous because of the entropy contribution to the Gibbs free energy, which makes the adsorption overall thermodynamically unfavorable. This balance of effects, computed here from molecular simulations, could be measured experimentally using high-pressure calorimetry, as it has been done for the purely siliceous silicalite-1 zeolite. $\frac{52153}{53}$

\subsection{Thermodynamics of ion entry into the nanopores}

In the previous sections, we described the behavior of intruded electrolytes in the pores of ZIF-8, using full periodic boundary conditions. Here, we want to investigate the thermodynamics of the process by which species (water molecules and ions) can actually enter the nanopores space, i.e. pass through the windows of the material and its external surface. We have thus modeled an explicit water/ZIF-8 interface, depicted in Figure 9; the system here thus contains both water in the bulk state in a $34 \AA \times 34 \AA \times 30 \AA$ reservoir, and water confined inside ZIF-8. We used the umbrella sampling simulations and the WHAM analysis method $\underline{54}$ to reconstruct the free energy profile of a single species $\left(\mathrm{Li}^{+}, \mathrm{Cl}^{-}\right.$, or $\left.\mathrm{H}_{2} \mathrm{O}\right)$ entering ZIF-8 


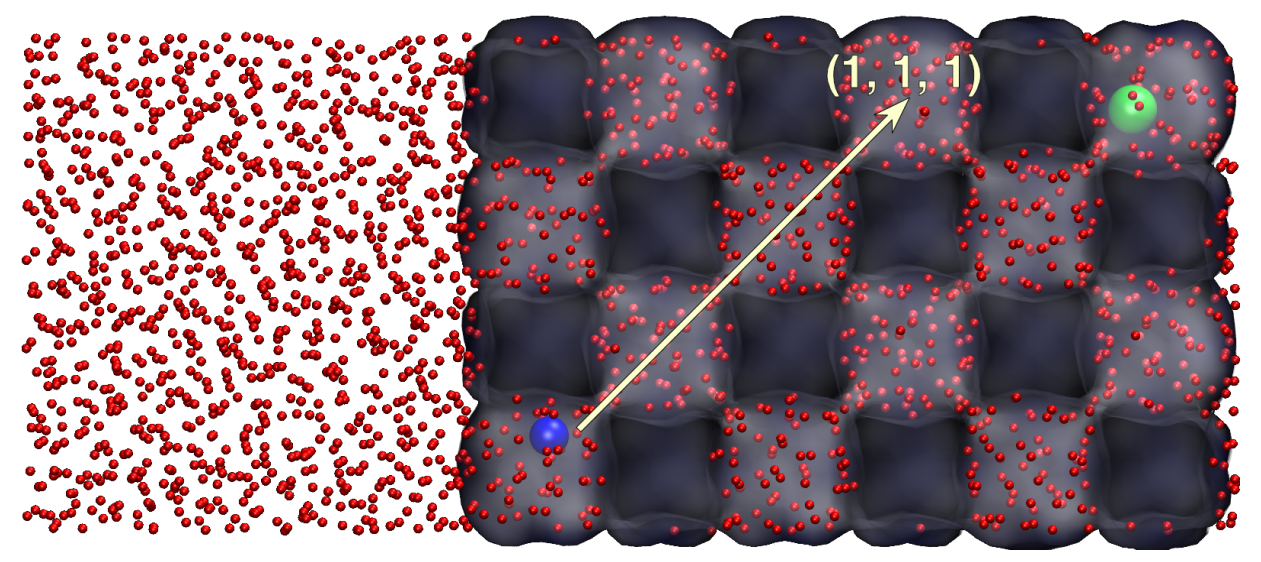

Figure 9: Representation of the system used for umbrella sampling simulation to compute the free energy profile of entry of lithium ions in ZIF-8. We used similar systems for the free energy profile of entry of chlorine and water. Water molecules are represented in red, lithium ions in pink and chlorine ion in green. ZIF-8 is represented as a transparent matrix.

along the (111) crystallographic axis. We placed the molecule of interest at $5 \AA$ of a window between the bulk and confined water, and the corresponding counter ion on the other side of the ZIF-8 slab (to keep the system neutral yet minimize ion-ion interactions). We ran a total of 121 umbrella sampling simulation for each species, spaced every $0.33 \AA$. Each simulation ran for $500 \mathrm{ps}$ in the $(N, V, T)$ ensemble, using the last step of the previous simulation as starting point. The resulting free energy profile is presented in Figure 10, together with the average number of neighbors at a given position on the axis (lower panel).

The first conclusion is that there is no free energy barrier for entry of the water molecule: the energy profile is flat and the number of neighbors is constant and around 4. This is coherent with the results already presented on the location of water molecules inside the ZIF-8 windows and on the number of neighbors for water molecules inside ZIF-8. It also confirms that the nature of the liquid-phase intrusion process is not a kinetic limitation of water adsorption, but actually due to thermodynamic hydrophobicity of the framework. For chlorine anions, we observe two barriers on the free energy profile, which correspond to the ZIF-8 windows at 0 and $15 \AA$. These barriers are correlated to a lower number of neighbors for the anion, dropping to a value of 4: there is not enough space inside the window to fit a chlorine ion and 7 water molecules, and the anion has to partially desolvate to pass through 

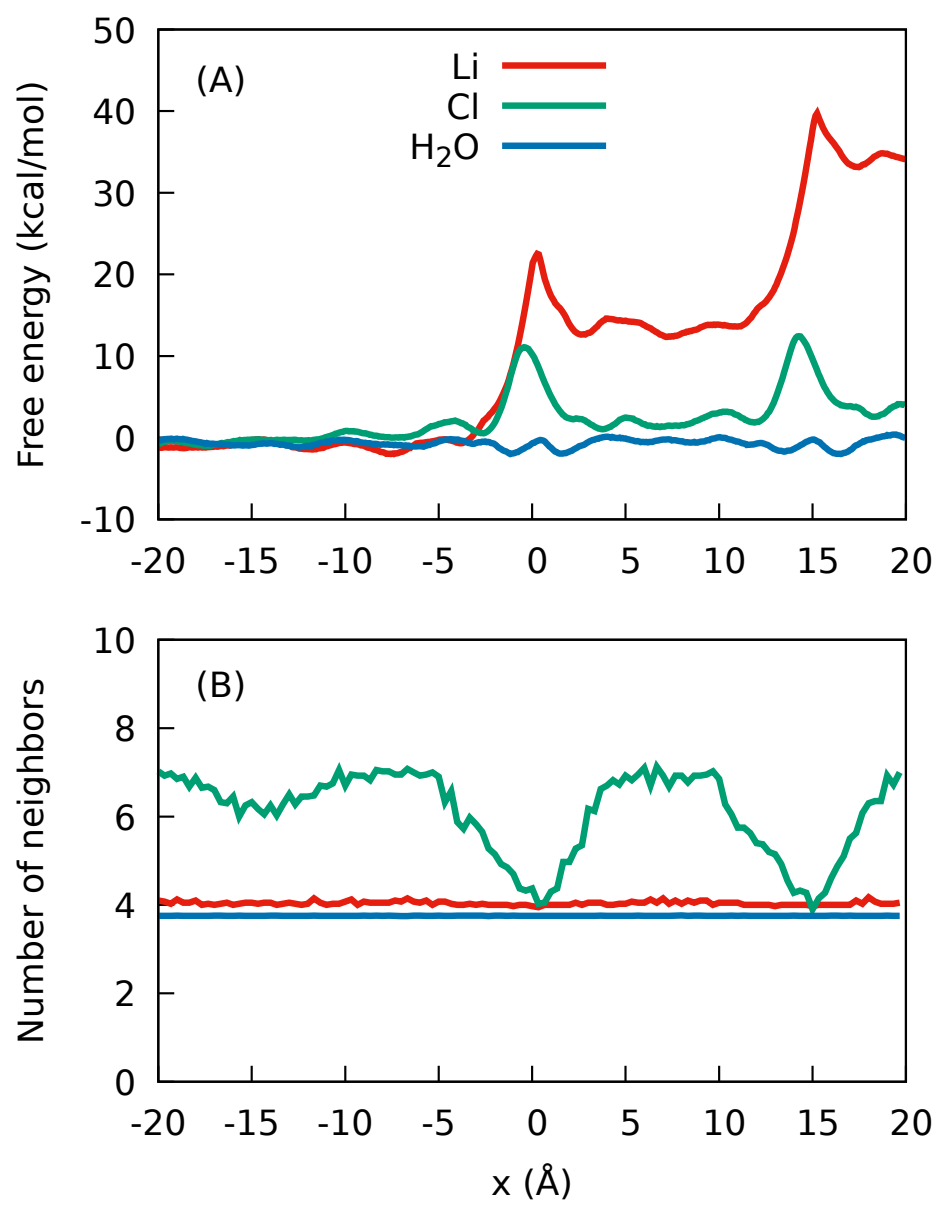

Figure 10: (A) Free energy profile of a single molecule entering ZIF-8 and (B) corresponding number of neighbors in the first solvation shell as function of the position of the molecule along the (111) crystallographic axis. The first ZIF-8 windows is at $x=0$; the $x<0$ area corresponds to bulk water, and the $x>0$ area to water-filled ZIF-8. We evaluated the uncertainty on the free energy profile using Monte Carlo bootstrapping,, 54 and found it to be at most $0.08 \mathrm{kcal} / \mathrm{mol}$ for $\mathrm{H}_{2} \mathrm{O}$, and $0.3 \mathrm{kcal} / \mathrm{mol}$ for $\mathrm{Cl}^{-}$and $\mathrm{Li}^{+}$.

the window - explaining the presence of the free energy barrier. Outside of these barrier, the profile is flat and at the same level as in the bulk liquid, meaning that while the entry of a single chlorine ion is a rare event, at long thermodynamic time scale, $\mathrm{Cl}$ ions should be able to enter in ZIF-8. Generally speaking, the $\mathrm{Cl}$ ions have a kinetic barrier to entry in the ZIF-8.

The results for Li are more surprising. We see both a high barrier at the first $(x=0 \AA)$ and second $(x=15 \AA$ ) windows; and a free energy difference between outside and inside the pores of roughly $13 \mathrm{kcal} / \mathrm{mol}$. This free energy difference is not only due to the bulk liquid 
to confined liquid transition, as it is also present in the transition between before and after the second window. At the same time, these barriers and energy differences are not linked to a difference in solvation as in the chlorine case, as the number of neighbors of lithium stays constant and around 4. Indeed, the solvation of $\mathrm{Li}^{+}$by water is much stronger, and its solvation sphere is smaller in size than $\mathrm{Cl}^{-}$. As lithium does not partially desolvate or rearrange to pass the barrier, the whole solvation sphere needs to go though a relatively small window, thus making the barrier higher. This points to a difference in nature between the $\mathrm{Li}^{+}$and $\mathrm{Cl}^{-}$ions, which will have to be probed further, for example by studies on other ions of different size.

From this $13 \mathrm{kcal} / \mathrm{mol}$ free energy difference, we can calculate a partition coefficient of $K=\exp (-\Delta F / k T) \simeq 410^{-10}$. This very small value means that there is, in practice, exclusion of the $\mathrm{Li}^{+}$ions from the nanopores — at infinite dilution. There may, however, be a collective effect when the concentration of $\mathrm{LiCl}$ increases, meaning we would get a very different value for a $\mathrm{LiCl}$ solution than for a single, isolated $\mathrm{Li}^{+}$cation.

\section{Conclusion}

Liquid intrusion of water and concentrated aqueous solutions in hydrophobic materials have been proposed for applications in mechanical energy storage and dissipation, and recently ZIF frameworks have been highlighted for the high energy density that they can store. However, while the process of intrusion has been well studied in various zeolitic materials over the last 20 years, there is relatively little information available on the behavior - at the microscopic scale - of water and electrolytes in hydrophobic metal-organic frameworks. These systems are difficult to probe experimentally, because liquid intrusion has to occur under high pressure. Therefore, we have used molecular dynamics simulations to shed some light onto the properties of $\mathrm{LiCl}$ aqueous solutions at various concentrations confined inside the pores of the ZIF-8 metal-organic framework. We show that the presence of the electrolyte has a moderate 
impact on the ZIF-8 framework, while the presence of the ZIF-8 matrix strongly influences the behavior of the confined aqueous solution, affecting the overall properties of the system. We also computed the free energy profile for the entry of water molecules and ions into the nanopores, showing a difference between anions and cations.

While this work provides an interesting picture of the $\mathrm{LiCl}$ electrolytes in $\mathrm{ZIF}-8$, it also opens a few venues for future research. The main one is the impact of the ion size on the properties of the confined liquid. Experiments have been performed with other ions of larger size, including $\mathrm{KCl}$, and there it is not even clear what fraction of the larger cations $\left(\mathrm{K}^{+}\right)$ actually can diffuse inside the nanopores. Computational approaches to these systems will be of great help in rationalizing the experimental results and provide a view of the microscopic mechanisms that are behind them.

Another one is to give a deeper look at the free energy barriers for ions passing through the windows of ZIF-8. While the windows are found, in the gas phase, to be very flexible and let diffuse molecules of large diameter (up to butane), we find that the entry of solvated species, such as ions in water, can be linked to a significant free energy barrier. Our free energy simulations of this process will have to be extended to other ions, in order to probe the influence of the size of both the ion and its solvation shell, but also to look at the influence of electrolyte concentration on the free energy profiles. Initial tests in this direction have shown that it should be technically possible, but convergence in such highly constrained systems is very difficult to achieve.

Finally, this work focused on the ZIF-8 framework, perhaps the most archetypal of the ZIF materials. The influence of framework functionalization with various imidazolate derivatives, which has shown to greatly impact adsorption in the gas phase, will surely also manifest itself in the liquid-phase intrusion processes. Relationships between nanoporous structure and intrusion properties have been studied in the past in hydrophobic zeolites, where the impact of structural flexibility does not greatly impact the intrusion behavior — except in cases of chemical reactivity between water and framework. Future work will need to address the 
question of the impact of the nanoporous host's flexibility on the intrusion-extrusion cycle: questions of both thermodynamics and kinetics are still widely open. In particular, whether one can fine-tune the intrusion of aqueous solutions in hydrophobic materials by control of flexibility is of large interest, since it would add another possible variable (in addition to pore size and solution concentration) to the dimensionality of the problem.

\section{Acknowledgement}

We acknowledge access to high-performance computing platforms provided by GENCI grant A0050807069.

\section{Supporting Information Available}

LAMMPS input files and initial configurations of the systems.

\section{References}

(1) Rouquerol, J.; Rouquerol, F.; Llewellyn, P.; Maurin, G.; Sing, K. S. Adsorption by powders and porous solids: principles, methodology and applications; Academic press, 2013.

(2) Wu, T.; Shen, L.; Luebbers, M.; Hu, C.; Chen, Q.; Ni, Z.; Masel, R. I. Enhancing the stability of metal-organic frameworks in humid air by incorporating water repellent functional groups. Chem. Commun. 2010, 46, 6120.

(3) Ghosh, P.; Kim, K. C.; Snurr, R. Q. Modeling Water and Ammonia Adsorption in Hydrophobic Metal-Organic Frameworks: Single Components and Mixtures. J. Phys. Chem. C 2013, 118, 1102-1110. 
(4) Wang, C.; Liu, X.; Keser Demir, N.; Chen, J. P.; Li, K. Applications of water stable metal-organic frameworks. Chem. Soc. Rev. 2016, 45, 5107-5134.

(5) Flanigen, E. M.; Bennett, J. M.; Grose, R. W.; Cohen, J. P.; Patton, R. L.; Kirchner, R. M.; Smith, J. V. Silicalite, a new hydrophobic crystalline silica molecular sieve. Nature 1978, 271, 512-516.

(6) Giaya, A.; Thompson, R. W.; Denkewicz Jr, R. Liquid and vapor phase adsorption of chlorinated volatile organic compounds on hydrophobic molecular sieves. Microporous Mesoporous Mater. 2000, 40, 205-218.

(7) Eroshenko, V.; Regis, R.-C.; Soulard, M.; Patarin, J. Energetics: A New Field of Applications for Hydrophobic Zeolites. J. Am. Chem. Soc. 2001, 123, 8129-8130.

(8) Soulard, M.; Patarin, J.; Eroshenko, V.; Regis, R. Stud. Surf. Sci. Catal.; Elsevier, 2004; Vol. 154; pp 1830-1837.

(9) Fraux, G.; Coudert, F.-X.; Boutin, A.; Fuchs, A. H. Forced intrusion of water and aqueous solutions in microporous materials: from fundamental thermodynamics to energy storage devices. Chem. Soc. Rev. 2017, 46, 7421-7437.

(10) Saada, M. A.; Soulard, M.; Marler, B.; Gies, H.; Patarin, J. High-Pressure Water Intrusion Investigation of Pure Silica RUB-41 and S-SOD Zeolite Materials. J. Phys. Chem. C 2010, 115, 425-430.

(11) Desbiens, N.; Demachy, I.; Fuchs, A. H.; Kirsch-Rodeschini, H.; Soulard, M.; Patarin, J. Water Condensation in Hydrophobic Nanopores. Angew. Chem. Int. Ed. 2005, 44, $5310-5313$.

(12) Humplik, T.; Raj, R.; Maroo, S. C.; Laoui, T.; Wang, E. N. Effect of Hydrophilic Defects on Water Transport in MFI Zeolites. Langmuir 2014, 30, 6446-6453. 
(13) Humplik, T.; Raj, R.; Maroo, S. C.; Laoui, T.; Wang, E. N. Framework water capacity and infiltration pressure of MFI zeolites. Microporous Mesoporous Mater. 2014, 190, 84-91.

(14) Ortiz, G.; Nouali, H.; Marichal, C.; Chaplais, G.; Patarin, J. Energetic performances of the metal-organic framework ZIF-8 obtained using high pressure water intrusionextrusion experiments. Phys. Chem. Chem. Phys. 2013, 15, 4888.

(15) Grosu, Y.; Gomes, S.; Renaudin, G.; Grolier, J.-P. E.; Eroshenko, V.; Nedelec, J.M. Stability of zeolitic imidazolate frameworks: effect of forced water intrusion and framework flexibility dynamics. RSC Adv. 2015, 5, 89498-89502.

(16) Michelin-Jamois, M.; Picard, C.; Vigier, G.; Charlaix, E. Giant Osmotic Pressure in the Forced Wetting of Hydrophobic Nanopores. Phys. Rev. Lett. 2015, 115.

(17) Ortiz, A. U.; Freitas, A. P.; Boutin, A.; Fuchs, A. H.; Coudert, F.-X. What makes zeolitic imidazolate frameworks hydrophobic or hydrophilic? The impact of geometry and functionalization on water adsorption. Phys. Chem. Chem. Phys. 2014, 16, 9940-9949.

(18) Ortiz, G.; Nouali, H.; Marichal, C.; Chaplais, G.; Patarin, J. Versatile Energetic Behavior of ZIF-8 upon High Pressure Intrusion-Extrusion of Aqueous Electrolyte Solutions. J. Phys. Chem. C 2014, 118, 7321-7328.

(19) Camblor, M. A.; Corma, A.; Valencia, S. Spontaneous nucleation and growth of pure silica zeolite- $\beta$ free of connectivity defects. Chem. Commun. 1996, 2365.

(20) Ryzhikov, A.; Khay, I.; Nouali, H.; Daou, T. J.; Patarin, J. Drastic change of the intrusion-extrusion behavior of electrolyte solutions in pure silica $*$ BEA-type zeolite. Phys. Chem. Chem. Phys. 2014, 16, 17893-17899.

(21) Arletti, R.; Ronchi, L.; Quartieri, S.; Vezzalini, G.; Ryzhikov, A.; Nouali, H.; Daou, T. J.; Patarin, J. Intrusion-extrusion experiments of $\mathrm{MgCl} 2$ aqueous solution in pure silica 
ferrierite: Evidence of the nature of intruded liquid by in situ high pressure synchrotron X-ray powder diffraction. Microporous Mesoporous Mater. 2016, 235, 253-260.

(22) Lee, Y.; Hriljac, J. A.; Vogt, T.; Parise, J. B.; Artioli, G. First Structural Investigation of a Super-Hydrated Zeolite. J. Am. Chem. Soc. 2001, 123, 12732-12733.

(23) Seoung, D.; Lee, Y.; Kao, C.-C.; Vogt, T.; Lee, Y. Super-Hydrated Zeolites: PressureInduced Hydration in Natrolites. Chem. Eur. J. 2013, 19, 10876-10883.

(24) Horike, S.; Shimomura, S.; Kitagawa, S. Soft porous crystals. Nature Chem. 2009, 1, 695-704.

(25) Schneemann, A.; Bon, V.; Schwedler, I.; Senkovska, I.; Kaskel, S.; Fischer, R. A. Flexible metal-organic frameworks. Chem. Soc. Rev. 2014, 43, 6062-6096.

(26) Haigis, V.; Coudert, F.-X.; Vuilleumier, R.; Boutin, A. Investigation of structure and dynamics of the hydrated metal-organic framework MIL-53(Cr) using first-principles molecular dynamics. Phys. Chem. Chem. Phys. 2013, 15, 19049.

(27) Boutin, A.; Bousquet, D.; Ortiz, A. U.; Coudert, F.-X.; Fuchs, A. H.; Ballandras, A.; Weber, G.; Bezverkhyy, I.; Bellat, J.-P.; Ortiz, G. et al. Temperature-Induced Structural Transitions in the Gallium-Based MIL-53 Metal-Organic Framework. J. Phys. Chem. C 2013, 117, 8180-8188.

(28) Coudert, F.-X.; Ortiz, A. U.; Haigis, V.; Bousquet, D.; Fuchs, A. H.; Ballandras, A.; Weber, G.; Bezverkhyy, I.; Geoffroy, N.; Bellat, J.-P. et al. Water Adsorption in Flexible Gallium-Based MIL-53 Metal-Organic Framework. J. Phys. Chem. C 2014, 118, 53975405.

(29) Mortada, B.; Chaplais, G.; Veremeienko, V.; Nouali, H.; Marichal, C.; Patarin, J. Energetic Performances of ZIF-8 Derivatives: Impact of the Substitution (Me, Cl, or Br) on Imidazolate Linker. J. Phys. Chem. C 2018, 122, 3846-3855. 
(30) Plimpton, S. Fast parallel algorithms for short-range molecular dynamics; 1993.

(31) Fiorin, G.; Klein, M. L.; Hénin, J. Using collective variables to drive molecular dynamics simulations. Mol. Phys. 2013, 111, 3345-3362.

(32) Berendsen, H. J. C.; Grigera, J. R.; Straatsma, T. P. The missing term in effective pair potentials. J. Phys. Chem. 1987, 91, 6269-6271.

(33) Zheng, B.; Sant, M.; Demontis, P.; Suffritti, G. B. Force Field for Molecular Dynamics Computations in Flexible ZIF-8 Framework. J. Phys. Chem. C 2012, 116, 933-938.

(34) Chowdhuri, S.; Chandra, A. Hydration structure and diffusion of ions in supercooled water: Ion size effects. J. Chem. Phys. 2003, 118, 9719-9725.

(35) Martínez, L.; Andrade, R.; Birgin, E. G.; Martínez, J. M. PACKMOL: A package for building initial configurations for molecular dynamics simulations. J. Comput. Chem. 2009, 30, 2157-2164.

(36) Marcus, Y. Ionic radii in aqueous solutions. Chem. Rev. 1988, 88, 1475-1498.

(37) Luzar, A.; Chandler, D. Hydrogen-bond kinetics in liquid water. Nature 1996, 379 , $55-57$.

(38) Fogarty, A. C.; Coudert, F.-X.; Boutin, A.; Laage, D. Reorientational Dynamics of Water Confined in Zeolites. ChemPhysChem 2014, 15, 521-529.

(39) Scatena, L. F.; Brown, M. G.; Richmond, G. L. Water at Hydrophobic Surfaces: Weak Hydrogen Bonding and Strong Orientation Effects. Science 2001, 292, 908-912.

(40) Jeffery, S.; Hoffmann, P. M.; Pethica, J. B.; Ramanujan, C.; Özer, H. O.; Oral, A. Direct measurement of molecular stiffness and damping in confined water layers. Phys. Rev. B 2004, 70, 216. 
(41) Romero-Vargas Castrillón, S.; Giovambattista, N.; Aksay, I. A.; Debenedetti, P. G. Evolution from Surface-Influenced to Bulk-Like Dynamics in Nanoscopically Confined Water. J. Phys. Chem. B 2009, 113, 7973-7976.

(42) Scalfi, L.; Fraux, G.; Boutin, A.; Coudert, F.-X. Structure and Dynamics of Water Confined in Imogolite Nanotubes. Langmuir 2018, 34, 6748-6756.

(43) Tazi, S.; Boţan, A.; Salanne, M.; Marry, V.; Turq, P.; Rotenberg, B. Diffusion coefficient and shear viscosity of rigid water models. J. Phys. Condensed Matter 2012, 24, 284117.

(44) Chaplais, G.; Fraux, G.; Paillaud, J.-L.; Marichal, C.; Nouali, H.; Fuchs, A. H.; Coudert, F.-X.; Patarin, J. Impacts of the Imidazolate Linker Substitution $(\mathrm{CH} 3, \mathrm{Cl}$, or $\mathrm{Br}$ ) on the Structural and Adsorptive Properties of ZIF-8. J. Phys. Chem. C 2018, 122, 26945-26955.

(45) Coudert, F.-X. Molecular Mechanism of Swing Effect in Zeolitic Imidazolate Framework ZIF-8: Continuous Deformation upon Adsorption. ChemPhysChem 2017, 18, 2732-2738.

(46) Fairen-Jimenez, D.; Moggach, S. A.; Wharmby, M. T.; Wright, P. A.; Parsons, S.; Düren, T. Opening the Gate: Framework Flexibility in ZIF-8 Explored by Experiments and Simulations. J. Am. Chem. Soc. 2011, 133, 8900-8902.

(47) Bennett, T. D.; Simoncic, P.; Moggach, S. A.; Gozzo, F.; Macchi, P.; Keen, D. A.; Tan, J.-C.; Cheetham, A. K. Reversible pressure-induced amorphization of a zeolitic imidazolate framework (ZIF-4). Chem. Commun. 2011, 47, 7983.

(48) Cao, S.; Bennett, T. D.; Keen, D. A.; Goodwin, A. L.; Cheetham, A. K. Amorphization of the prototypical zeolitic imidazolate framework ZIF-8 by ball-milling. Chem. Commun. 2012, 48, 7805 .

(49) Ortiz, A. U.; Boutin, A.; Fuchs, A. H.; Coudert, F.-X. Investigating the Pressure-Induced 
Amorphization of Zeolitic Imidazolate Framework ZIF-8: Mechanical Instability Due to Shear Mode Softening. J. Phys. Chem. Lett. 2013, 4, 1861-1865.

(50) Tan, J.-C.; Civalleri, B.; Lin, C.-C.; Valenzano, L.; Galvelis, R.; Chen, P.-F.; Bennett, T. D.; Mellot-Draznieks, C.; Zicovich-Wilson, C. M.; Cheetham, A. K. Exceptionally Low Shear Modulus in a Prototypical Imidazole-Based Metal-Organic Framework. Phys. Rev. Lett. 2012, 108.

(51) Lanman, E. H.; Mair, B. J. The Compressibility of Aqueous Solutions. J. Am. Chem. Soc. 1934, 56, 390-393.

(52) Karbowiak, T.; Paulin, C.; Ballandras, A.; Weber, G.; Bellat, J.-P. Thermal Effects of Water Intrusion in Hydrophobic Nanoporous Materials. J. Am. Chem. Soc. 2009, 131, 9898-9899.

(53) Karbowiak, T.; Paulin, C.; Bellat, J.-P. Determination of water intrusion heat in hydrophobic microporous materials by high pressure calorimetry. Microporous Mesoporous Mater. 2010, 134, 8-15.

(54) Grossfield, A. WHAM: the weighted histogram analysis method, version 2, http://membrane.urmc.rochester.edu/content/wham. 


\section{TOC Graphic}

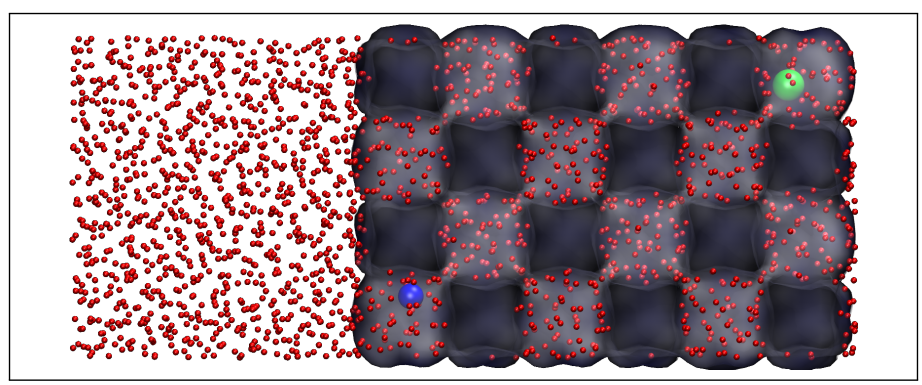

